J. Lake Sci. (湖泊科学), 2013, 25(4): 593-599

http: //www.jlakes.org. E-mail : jlakes@niglas.ac.cn

(C) 2013 by Journal of Lake Sciences

\title{
雷达测雨误差及其对淮河流域径流模拟的影响”
}

\author{
叶金印 ${ }^{1,2}$, 高玉芳 ${ }^{3,4}$, 李致家 ${ }^{1}$ \\ (1: 河海大学水文水资源学院,南京 210098) \\ (2:淮河流域气象中心,蚌埠 233040$)$ \\ (3: 南京信息工程大学江苏省农业气象重点实验室,南京 210044) \\ (4:南京信息工程大学应用气象学院,南京 210044)
}

摘 要: 将雷达测雨数据与分布式水文模型相耦合进行径流过程模拟, 分析雷达测雨误差及其径流过程模拟效果, 研究 雷达测雨误差对径流过程模拟的影响效应. 在对淮河流域气象中心业务化的 5 种淮河流域雷达测雨数据进行误差分析的 基础上,采用雷达测雨数据驱动 HEC-HMS 水文模型,模拟分析淮河息县水文站以上流域 2007 年 7 月 $1-10$ 日强降雨集 中期的径流过程. 结果表明:利用雷达测雨数据的径流模拟结果与实测资料的模拟结果基本吻合,各种雷达测雨数据误 差经过 HEC-HMS 水文模型传递后, 误差明显减小. 联合校准法对应的模拟效果最好,过程流量相对误差 $N B s^{\prime}$ 和洪峰流量 相对误差 $Z^{\prime}$ 分别为 $-20.2 \%$ 和 $-13.3 \%$.

关键词: 雷达测雨; 水文模型; 径流模拟;误差传递;淮河流域

\section{Radar-rainfall errors and their effect on runoff simulation in Huaihe River basin}

YE Jinyin ${ }^{1,2}$, GAO Yufang ${ }^{3,4}$ \& LI Zhijia ${ }^{1}$

(1: College of Hydrology and Water Resources, Hohai University, Nanjing 210098, P. R. China)

(2: Huaihe River Basin Meteorological Center, Bengbu 233040, P. R. China)

(3: Jiangsu Key Laboratory of Agricultural Meteorology, Nanjing University of Information Science and Technology, Nanjing 210044, P. R. China)

(4: School of Applied Meteorology, Nanjing University of Information Science and Technology, Nanjing 210044, P. R. China)

Abstract: Coupling radar-rainfall data with distributed hydrological model was important in improving flood forecast precision and leading time. However, radar-rainfall error propagation in hydrological model largely affected runoff process simulation. In this study, the operational radar-rainfall data, provided by Huaihe River basin Meteorological Center, was analyzed. With HEC-HMS hydrological model input, radar-rainfall data was used to simulate and analyze the runoff process of rainfall concentration period, in the Xixian hydrological station of Huaihe River basin, during July $1-10$, 2007. The results showed that runoff simulation based on radar-rainfall data was consistent with the simulation based on rain gauge data. The radar-rainfall input error had been decreased obviously after the propagation of the HEC-HMS model. Runoff simulation based on radar-rainfall data by union calibration was the best, whose relative error were $-20.2 \%$ and $-13.3 \%$ for flow process and peak flow, respectively.

Keywords: Radar-rainfall estimation; hydrological model; runoff simulation; error propagation; Huaihe River basin

降水的选择是水文模拟中一个至关重要的因素,往往比水文模型的选择更重要 ${ }^{[1]}$. 由雷达回波反演的 降水资料, 不仅时空分辨率较高, 而且可以获得流域尺度上降雨的空间分布特征, 其空间分布明显要好于雨 量站网的信息采集方式. 因此,雷达测雨及其在水文模型中的应用受到越来越多的关注 ${ }^{[2-5]}$. 尤其是近些年 来, 分布式水文模型的迅速发展进一步推动了雷达测雨技术在水文预报中的应用 ${ }^{[6]}$. 但另一方面,受环境、

* 国家自然科学基金项目 (40875012,40905019)、国家自然科学基金青年项目 (41105077)、江苏省自然科学基金面 上项目 (SBK201222411) 和公益性行业 (气象) 科研专项项目 (GYHY201006037) 联合资助. 2012-09-20 收稿; 2013-01-05 收修改稿. 叶金印, 男,1968 年生,博士研究生, 高级工程师; E-mail : yejinyin@ sina. com. 
算法及雷达本身的限制, 由雷达回波反演的降水也存在一定的误差和不确定性. 雷达技术自身的复杂性以 及降雨-径流转化过程中对水文模型的选择、参数的调试和计算、流域特征的数量表达等难以把握, 会影响 水文预报效果.

目前, 国内外将雷达测雨数据与分布式水文模型相结合的研究已有一些成果 ${ }^{[7-9]}$, 雷达测雨误差经过径 流模拟后,会因研究区域位置、风暴特征等因素放大或缩小 ${ }^{[10-11]}$. 另外,分布式水文模型的应用效果主要依 赖于集水区类型、输人数据质量 ${ }^{[12]}$ 和模型自身特点 ${ }^{[13]}$ 等. 不同分布式水文模型根据研究区域时空分布及预 报变量 (如径流量、流域和水库水位、土壤湿度等) 有不同的应用. 对于每一种应用, 模型结构及复杂程度会 影响雷达测雨误差对径流模拟的效应. 在水文模拟中降雨输人误差和模型误差都会通过模拟过程加以传 递, 降雨量的数据误差是水文模型不确定性的重要来源, 因此, 雷达测雨误差及其对径流模拟的影响效应研 究是雷达测雨资料应用于洪水预报的关键部分, 对于进一步认识水文变化规律、提供科学合理的决策支持 有着较大的现实意义.

本文采用标准化偏估 ( $N B$, Normalized Bias) 指标对基于 $\mathrm{Z}-\mathrm{I}$ 关系以及采用平均校准 (Average)、最优校 准 (Optimal)、联合校准 (Union) 和卡尔曼滤波校准 (Kalman) 等校准方法的淮河流域气象中心 5 种雷达测雨 数据进行误差分析. 再分别利用 5 种雷达测雨数据驱动 HEC-HMS 水文模型, 研究雷达测雨误差对径流过程 模拟的影响.

\section{1 数据与方法}

\section{1 雷达测雨数据来源}

以淮河流域气象中心业务化运行的淮河流域雷达定量降水估算系统所形成的 5 种雷达测雨数据产品 为数据源. 该系统利用淮河流域内 6 部 $\mathrm{S}$ 波段新一代天气雷达数据和高密度自动雨量站观测资料, 分别采 用 $\mathrm{Z}-\mathrm{I}$ 关系, 以及平均校准、最优校准、联合校准和卡尔曼滤波校准等方法进行降水的估测和校准, 实现淮 河流域定量降水的估测 ${ }^{[14]}$. 其中, $Z-I$ 关系法是利用雷达反射率因子 $Z$ 和降水强度 $I$ 之间的理论关系进行 雨量估算, 得到的雨量估算值就是未经订正的雷达测雨值. 平均校准法是指在整个区域内, 统计地面降水量 观测值和未经订正的相应雷达估算值之间的平均差异, 并以此作为校准因子来进行雨量估测校准. 卡尔曼 滤波校准法, 首先假设降水估算值是由降水真值、系统误差以及随机误差 3 部分组成; 并认为降水估算值可 以通过对独立估计值进行一种加权平均而得到; 最后, 通过选择合适的权重因子使得平均结果具有最小 方差, 这个平均结果就是由卡尔曼滤波校准法得到的降水估算值. 最优插值校准法是一种在均方差最小 条件下的最优线性插值方法, 先在有地面雨量观测站点的地方, 用雨量观测值对雷达测雨值进行订正, 然 后通过最优插值订正得到雷达测雨值. 联合校准法是综合卡尔曼滤波校准法和最优插值校准法的一种校 准方法.

数据的空间分辨率为 $1 \mathrm{~km} \times 1 \mathrm{~km}$, 时间间隔为 $1 \mathrm{~h} .6$ 部雷达分别位于郑州、驻马店、阜阳、合肥、南京和 徐州,具体分布如图 1 所示.

\section{2 雷达测雨数据误差分析方法}

以雨量站观测数据为参照, 采用标准化偏估 $N B^{[15]}$ 指标对雷达测雨的降水量偏差进行精度分析, 计算公 式为:

$$
N B=\frac{\sum_{i=1}^{N}\left(R_{i}-G_{i}\right)}{\sum_{i=1}^{N} G_{i}} 100 \%
$$

式中, $N$ 为观测事件数, $G_{i}$ 为雨量站观测数据 $(\mathrm{mm} / \mathrm{h}), R_{i}$ 为对应的测雨雷达估测值 $(\mathrm{mm} / \mathrm{h})$.

\section{3 研究区域概况及水文模型的建立}

息县水文站是淮河上游干流重要的控制站. 本文以淮河息县水文站以上流域为研究区域, 流域面积为 $10190 \mathrm{~km}^{2}$, 去除南湾、石山口两座大型水库面积后是 $8826 \mathrm{~km}^{2}$. 流域内地形呈现西高东低趋势, 高程范围在 $15 \sim 839 \mathrm{~m}$ 之间, 平均高程 $142 \mathrm{~m}$. 流域辖区的中山区及低山区、丘林区, 主要土壤类型为黄棕壤土, 土体有明 显的黏化过程, 呈酸性和微酸性, 生长有松、衫等针、阔叶林及漆树、油桐、油茶等经济林木. 在中山、低山、岗 丘及河谷等各种地貌类型农田内广泛分布着水稻土类, 主要种植稻、麦、油菜等农作物. 淮河息县站以上流 


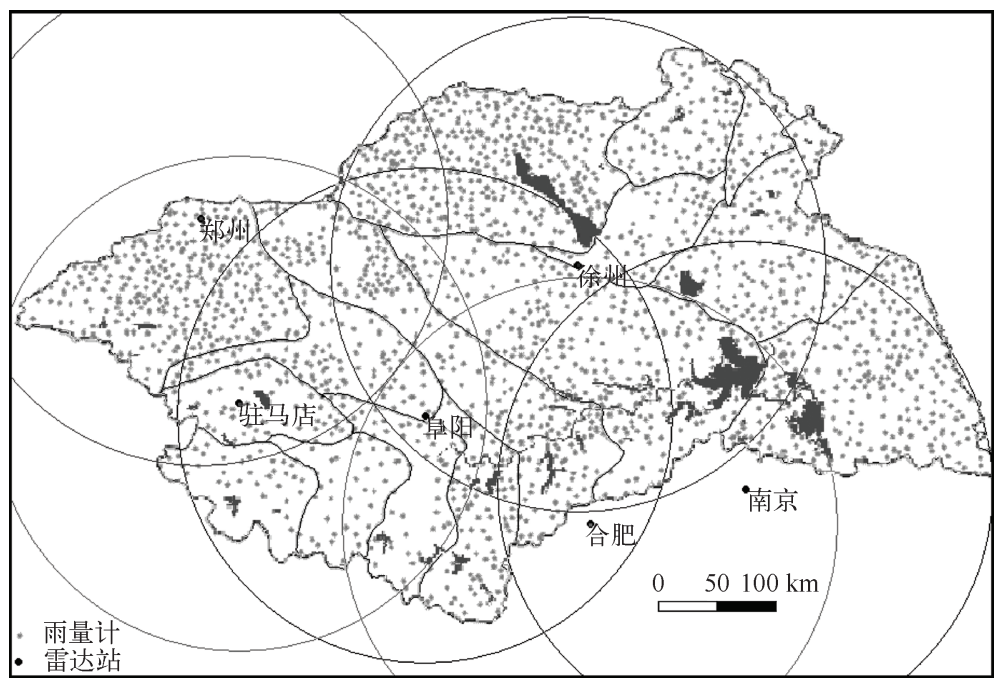

图 1 淮河流域 6 部雷达、雨量计分布及覆盖区域

Fig. 1 Distribution of 6 radars and rain gauges in Huaihe River basin

域水系及子流域划分见图 2.

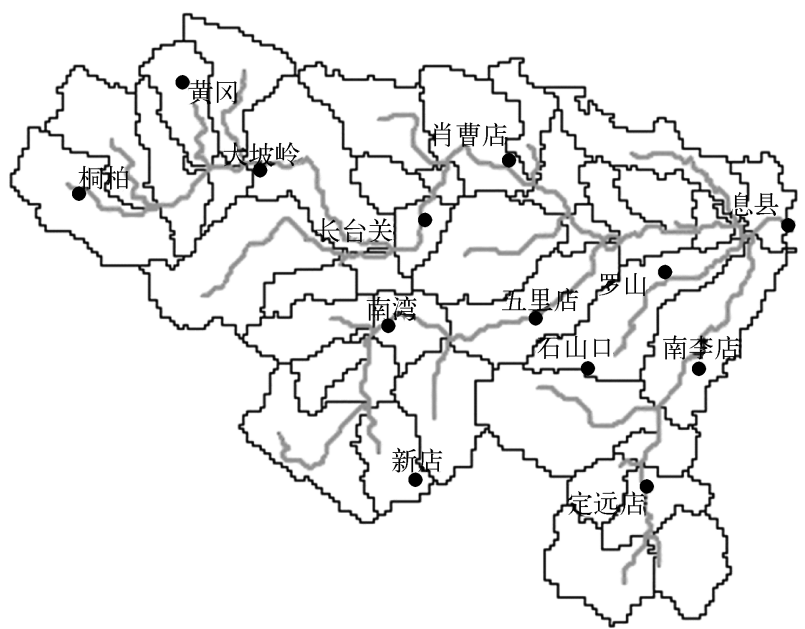

图 2 淮河息县站以上流域水系及子流域划分

Fig. 2 The stream and sub-basins over Xixian hydrological station of Huaihe River basin

选用 HEC-HMS 水文模型进行径流模拟 ${ }^{[16-17]}$. HEC-HMS 模型采用地理信息系统技术对流域进行各项分 析和各种参数的提取, 在模型系统中实现各种方法的模块化, 在产流和汇流过程中, 可根据研究区域的情 况,实现不同方法之间的组合, 从而得到研究区水文过程模拟的最佳方法. 初损后损模型简单易用,故选其 作为产流模型. 另外, 采用 SCS 模型作为直接径流汇流模型, 采用指数退水模型作为地下水模型, 采用马斯 京根法进行河道汇流演算.

\section{4 径流模拟误差分析方法}

利用过程流量百分比误差、洪峰流量百分比误差 ${ }^{[18]}$ 分析模拟径流与实测径流的相对精度. 


$$
\begin{aligned}
& N B s=\frac{\sum_{i=1}^{N}\left(Q_{\text {sim }}(i)-Q_{\text {obs }}(i)\right)}{\sum_{i=1}^{N} Q_{\text {obs }}(i)} 100 \% \\
& N B s^{\prime}=\frac{\sum_{i=1}^{N}\left(Q_{\text {sim }}(i)-Q(i)\right)}{\sum_{i=1}^{N} Q(i)} 100 \%
\end{aligned}
$$

式中, $N B s 、 N B s^{\prime}$ 为过程流量误差, $Q_{\text {sim }}(i) 、 Q_{\mathrm{obs}}(i) 、 Q(i)$ 分别为第 $i$ 时刻基于雷达测雨数据的模拟流量、观测 流量和基于雨量站观测数据的模拟流量.

$$
\begin{aligned}
& Z=\left|\frac{Q_{\text {obs }}(\text { peak })-Q_{\text {sim }}(\text { peak })}{Q_{\text {obs }}(\text { peak })}\right| 100 \% \\
& Z^{\prime}=\left|\frac{Q(\text { peak })-Q_{\text {sim }}(\text { peak })}{Q(\text { peak })}\right| 100 \%
\end{aligned}
$$

式中, $Z 、 Z^{\prime}$ 为洪峰流量误差, $Q_{\mathrm{obs}}$ ( peak) 为实测洪峰流量, $Q_{\mathrm{sim}}$ ( peak) 为基于雷达测雨数据的模拟洪峰流量, $Q_{\text {(peak) }}$ 为基于雨量站观测数据的模拟洪峰流量.

$N B s^{\prime}$ 和 $Z^{\prime}$ 指标去除了模型本身对径流模拟的影响.

\section{2 雷达测雨误差分析}

利用息县以上流域共 20 个雨量站的实测逐时降雨资料为参照, 对 2007 年 7 月 $1-10$ 日降雨集中期 5 种雷达测雨数据进行误差分析. 从雨量站实测与雷达估测 $1 \mathrm{~h}$ 累积降雨量对比图 (图 3 ) 可以看出, 在降雨量 为 $2 \sim 10 \mathrm{~mm} / \mathrm{h}$ 时, 这几种校准方法一致是低估, Average 校准方法低估程度最小, Kalman 校准方法低估最明 显. 降雨量为 $0 \sim 2 \mathrm{~mm} / \mathrm{h}$ 及大于 $10 \mathrm{~mm} / \mathrm{h}$ 时, 这几种校准方法高估、低估现象都存在. 通过对区域内降水强 度随高度的垂直分布廓线 ${ }^{[19]}$ 分析, 可以发现在 $6 \mathrm{~km}$ 高度以下降水强度有随着高度的降低呈增加的趋势. 由 于雷达探测到的是空中的雨水信息而非地面的降水信息, 因此降水随高度增加而减少的特征是引起雷达测 雨结果普遍偏低的主要原因.

从 2007 年 7 月 1-10 日降雨集中期 5 种雷达测雨数据的标准化偏估统计 (表 1 ) 可以看出, Average、Optimal、Union 和 Kalman 四种校准方法估测的 $1 \mathrm{~h}$ 降雨量都接近雨量站观测值. Union 校准方法估测的 $1 \mathrm{~h}$ 降 雨量 $N B$ 值最低, Average 和 Optimal 校准方法次之, Kalman 校准方法最差. Union 校准方法与其他估测和校 准方法相比, $N B$ 值的平均值 (MEAN)、基于样本估算标准偏差( STDEV)、最小值( MIN)、最大值( MAX)更接 近理想值, 可以看出雷达估算降水经校准后, 明显提高了估测精度.

表 1 雷达测雨数据标准化偏估统计结果 *

Tab. 1 NBs of radar rainfall estimation results with 5 methods

\begin{tabular}{ccccccc}
\hline & 统计参数 & $\mathrm{Z}-\mathrm{I}$ & Optimal & Average & Kalman & Union \\
\hline \multirow{2}{*}{$N B$} & MEAN & $-45.9 \%$ & $-35 \%$ & $-38.1 \%$ & $-32.5 \%$ & $-31.9 \%$ \\
& STDEV & $-10.9 \%$ & $-8.9 \%$ & $-10.2 \%$ & $-7.9 \%$ & $-7.2 \%$ \\
& MIN & $-80.1 \%$ & $-70.8 \%$ & $-72.6 \%$ & $-71.3 \%$ & $-68.6 \%$ \\
& MAX & $-23.2 \%$ & $-27.3 \%$ & $-22 \%$ & $-21.4 \%$ & $-20.4 \%$ \\
\hline
\end{tabular}

* Z - I、Optimal Average、Kalman、Union 分别为 Z - I 关系、平均校准、最优校准、联合校准和卡尔曼滤波校准等方法的雷达 测雨数据.

\section{HEC-HMS 模型参数率定及径流模拟误差分析}

\section{1 模型参数率定}

本文采用的 DEM 数据是由美国太空总署 (NASA) 和国防部国家测绘局 (NIMA) 联合测量的 SRTM 


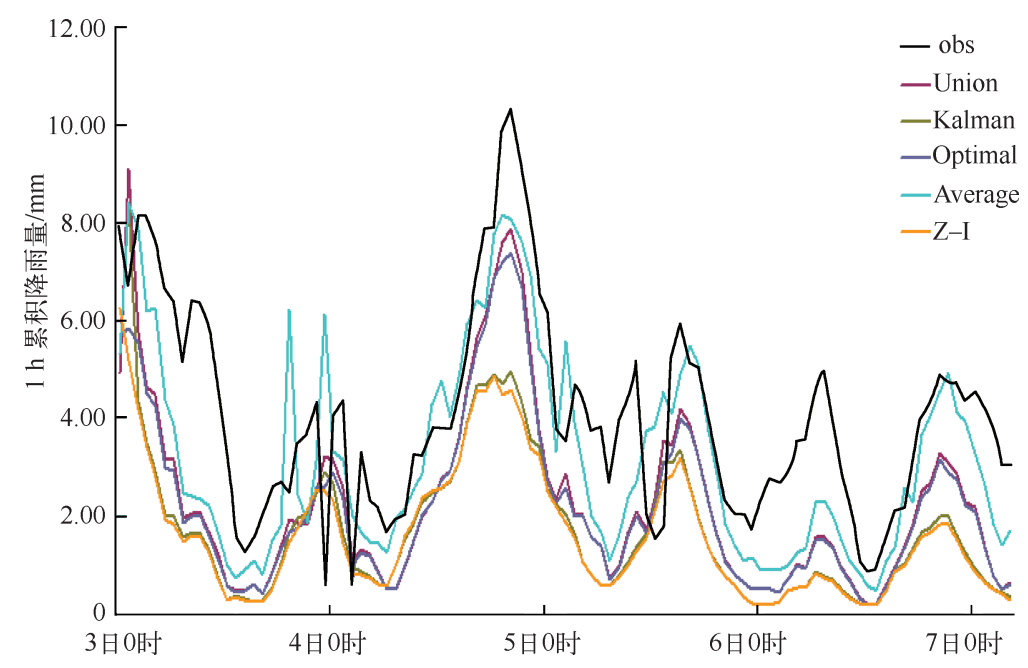

图 3 雨量站实测及雷达估测 $1 \mathrm{~h}$ 累积降雨量

Fig. 3 Time serial of $1 \mathrm{~h}$ rainfall from rain gauge and radar

(Shuttle Radar Topography Mission) $90 \mathrm{~m}$ 分辨率的原始高程数据. 数据来源于中国科学院计算机网络信息中 心国际科学数据镜像网站 (http://datamirror. csdb. cn).

在对研究区域水系进行子流域划分的基础上,采用 2005 年 4-9 月的水文气象资料进行 HEC-HMS 模 型的参数率定. 在率定模型参数基础上,采用 2007 年 7 月 12-14 日、8 月 2-20 日的 2 次降水过程的逐时资 料, 将息县站洪峰流量误差、过程流量误差控制在 $15 \%$ 以内 ${ }^{[20]}$, 流量峰现时间误差控制在 1 个时段 $(6 \mathrm{~h})$ 以 内,通过迭代计算 ${ }^{[21]}$ 对模型进行校准.

\section{2 径流模拟分析}

将 2007 年 7 月 $1-10$ 日强降雨集中期的 5 种雷达测雨数据作为 HEC-HMS 水文模型的输人进行水文模 拟,对观测径流与模拟径流的对比结果可以看出, Union 校核的雷达测雨数据对应的径流模拟结果与实测径 流最为接近, Z-I 关系的雷达测雨数据对应的径流模拟结果最差 (图 4). 另外, 2007 年 7 月 2 日 20:00 洪峰 没有模拟出来,而对于后面相对较大的洪峰模拟效果比较好.

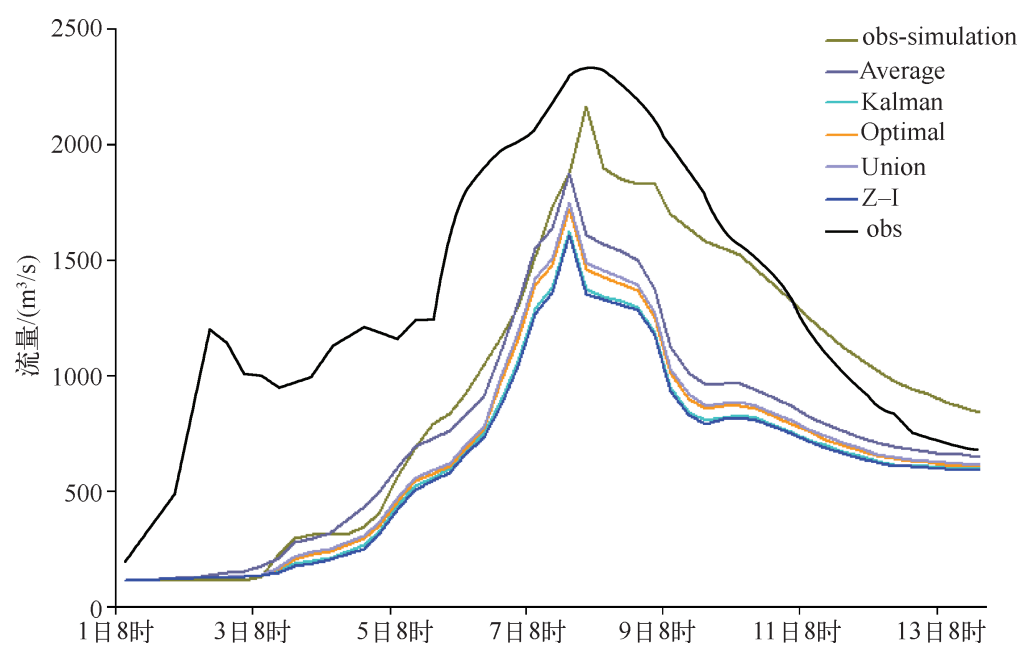

图 4 基于雨量站测雨和雷达测雨数据的 HEC-HMS 模型径流过程

Fig. 4 The flow processes based on rain gauge and radar-rainfall 
对 5 种雷达测雨数据驱动 HEC-HMS 水文模型的径流模拟结果进行统计, 表明雷达测雨数据作为 HEC-HMS模型输人的模拟径流对应的 NBs 值为负 (图 5 ), 这种结果与前面分析雷达数据存在低估相一致. 从地面雨量站降雨观测数据对应的径流模拟 (obs-simulation) $N B s 、 Z$ 值可以看出, 地面降雨观测数据作为 HEC-HMS模型输人的径流模拟存在低估现象, 其径流过程流量低估 $12.6 \%$, 洪峰流量低估 $7.5 \%$. 将各雷达 估测降雨数据的 $N B$ 值与径流模拟对应 $N B s 、 Z$ 值进行对比发现, $N B$ 值经过 HEC-HMS 模型传递后, $Z$ 值都 有减小, 且减小趋势较一致, Optimal Average、Kalman 校核方法对应径流模拟 NBs 值变化不大, 而 Union 和 $\mathrm{Z}-\mathrm{I}$ 关系方法对应的径流模拟 $N B s$ 增大, 尤其是 $\mathrm{Z}-\mathrm{I}$ 方法对应的 $N B$ 值最大为 $-45.9 \%, N B s$ 增幅也较大. 去除 HEC-HMS 模型本身对径流模拟的影响, 将雷达测雨数据的标准偏差 $N B$ 值与径流预报的标准偏差 $N B s^{\prime} 、 Z^{\prime}$ 值进行对比, 可以看出雷达测雨数据的 $N B$ 值通过水文模型传递后都有所减少. 其中, Union 校核方 法对应的径流模拟 $N B s^{\prime} 、 Z^{\prime}$ 值最小, 分别为 $-20.2 \% 、-13.3 \%$.

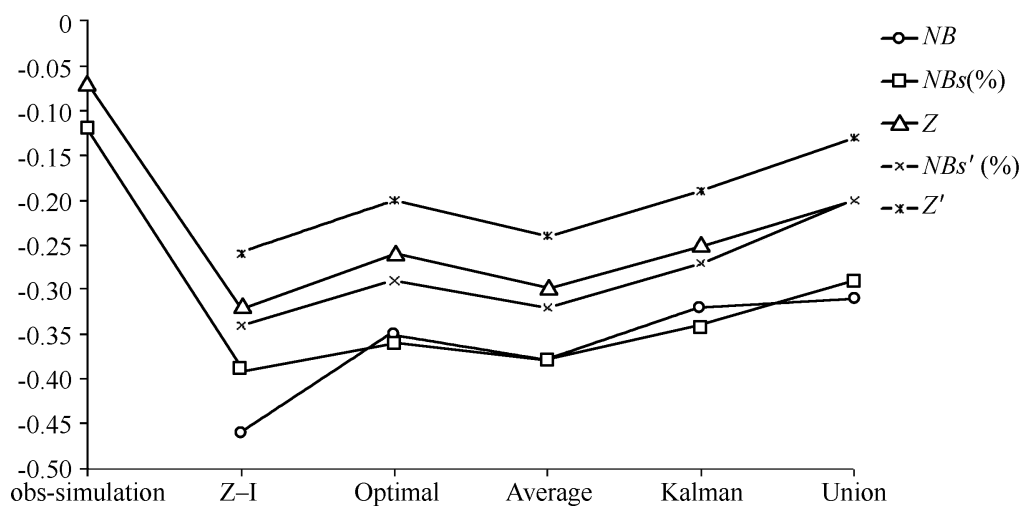

图 5 基于雷达测雨数据的径流模拟结果统计指标

Fig. 5 Statistical indicators for runoff simulation with radar-rainfall

\section{4 结论}

分析淮河流域气象中心业务化的淮河流域雷达测雨数据误差, 并用雷达测雨数据驱动 HEC-HMS 水文 模型, 研究雷达测雨误差对径流模拟的影响效应, 结果表明:

1 ) 雷达测雨数据 $N B$ 值与径流模拟的过程流量误差 $N B s$ 和洪峰流量误差 $Z$ 呈正相关, 说明减小雷达测 雨数据的 $N B$ 值, 对改进基于雷达测雨数据的径流模拟效果有重要意义.

2 ) 雷达测雨误差 $N B$ 值经过 HEC-HMS 水文模型传递后, 过程流量误差 $N B s^{\prime}$ 和洪峰流量误差 $Z 、 Z^{\prime}$ 都明 显减少, 过程流量误差 $N B s$ 对于 5 种雷达数据表现不同. 这一结论与已有研究 ${ }^{[22]}$ 结论一致, 进一步证明了雷 达测雨误差在不同研究区域,结合不同水文模型,对径流模拟会产生不同的影响趋势及效应.

3 ) 相比其他雷达测雨数据, 经过 Union 校准后的雷达测雨数据作为 HEC-HMS 模型输人, 径流模拟效果 有明显提高,过程流量误差 $N B s^{\prime}$ 和洪峰流量误差 $Z^{\prime}$ 明显较小.

4) 基于 5 种雷达测雨数据的径流过程和基于雨量站降雨观测数据的径流过程相比, 雷达测雨数据不仅 可以定量捕捉降雨的时空分布结构, 而且用雷达测雨数据驱动水文模型进行径流过程的模拟也具有一定的 精度. 将多源降水监测数据和雷达测雨数据相融合, 提高对降水的监测能力, 进而可以提高对水文过程的模 拟效果, 同时是值得我们未来探索的方向.

\section{5 参考文献}

[ 1 ] Wilk J, Kniveton D, Andersson L et al. Estimating rainfall and water balance over the Okavango River Basin for hydrological application. Journal of Hydrometeorology, 2006, 331:18-29.

[ 2 ] Artan G, Gadin H, Smith JL et al. Adequacy of satellite-derived rainfall data for streamflow modeling. Natural: Hazards, $2007,43: 167-185$. 
[ 3 ] Collischonn BW, Collischonn CE, Morelli T. Daily hydrological modeling in the Amazon basin using TRMM rainfall estimators. Journal of Hydrometeorology, 2008, 360 : 207-216.

[ 4 ] Su F, Hong Y, Lettenmaier DP. Evaluation of TRMM Multisatellite Precipitation Analysis(TMPA) and its utility in hydrologic prediction in the La Plata basin. Journal of Hydrometeorology, 2008,9:622-640.

[ 5 ] Beighley RE, Eggen KG, Dunne T et al. Simulating hydrologic and hydraulic processes throughout the Amazon River Basin. Hydrological Processes, 2009, 23:1221-1235.

[ 6 ] Berenguer M, Corral C, Sanchez-Diezma R. Hydrological validation of a radar-based nowcasting technique. Journal of Hydrometeorology, 2005, 6:532-550.

[7] 李致家,刘金涛,葛文忠等. 雷达估测降雨与水文模型的耦合在洪水预报中的应用. 河海大学学报,2004,32 (6): 601-606.

[ 8 ] 刘晓阳,毛节泰,李纪人等. 雷达估测降水模拟史灌河流域径流. 北京大学学报: 自然科学版,2002,(3):342-349.

[ 9 ] Gourley JJ, Giangrande SE, Hong Y. Impacts of polarimetric radar observations on hydrologic simulation. Journal of Hydrometeorology, 2010,6:781-797.

[10] Seo BC, Krajewski WF. Scale dependence of radar rainfall uncertainty : initial evaluation of nexrad's new super-resolution data for hydrologic applications. Journal of Hydrometeorology, 2010,11 : 1191-1198.

[11] Vivoni ER, Entekhabi D, Hoffman RN et al. Error propagation of radar rainfall nowcasting fields through a fully distributed flood forecasting model. Journal of Applied Meteorology and Climatology,2006,46:932-940.

[12] Wilk J, Kniveton D, Andersson L et al. Estimating rainfall and water balance over the Okavango River Basin for hydrological application. Journal of Hydrometeorology, 2006,331 : 18-29.

[13] Collier CG. On the propagation of uncertainty in weather radar estimates of rainfall through hydrological models. Meteorological Application, 2009,16:35-40.

[14] 黄 勇,胡 雯,何永健等. 多部雷达联合估算淮河流域降水.气象科学,2010,30(2):268-273.

[15] Developmental Tested Center. Model Evaluation Tools Version 3.0 (METv3.0). Boulder, Colorado, USA. Oct 2010 : c-1. http://www. dtcenter. org/met/users.

[16] USACE-HEC. Hydrologic modeling system HEC-HMS technical reference manual. US Army Corps of Engineers, Hydrologic Engineering Centre( HEC), Davis, USA. 2000.

[17] USACE-HEC. Hydrologic modeling system HEC-HMS v3.2 user's manual. US Army Corps of Engineers, Hydrologic Engineering Center(HEC), Davis, USA. 2008.

[18] Hydrologic Modeling System HEC-HMS, User's Manual Version 2.1: US Army Corps of Engineers Hydrologic Engineering Center, January 2001.

[19］傅云飞,宇如聪, 崔春光等. 基于热带测雨卫星探测的东亚降水云结构特征的研究. 暴雨灾害,2007,26(1):9-20.

[20] Wong S, Tang B, van Horen B. Strategic urban management in China: A case study of Guangzhou development district. Habitat International, 2006,30 (3) :645-667.

[21] HEC-HMS Technical Reference Manual : US Army Corps of Engineers Hydrologic Engineering Center, March 2000.

[22] 张利平, 张晓琳,徐 霞等. 基于水文模型的雷达监测降雨量误差传递研究. 水文,2012,32(1):13-18. 